\title{
Sterne und Zeichen
}

\section{B. Gurtner}

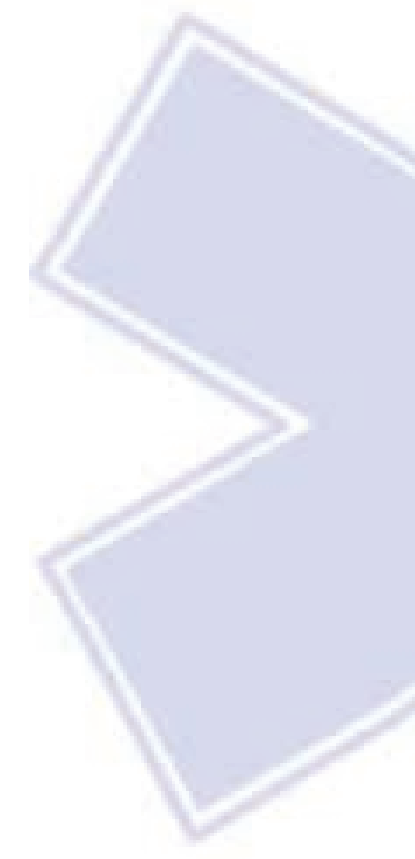

Korrespondenz:

Dr. med. Bernhard Gurtner

Eggstrasse 76

CH-8620 Wetzikon
Alles, was mit Lebensrettung oder erster Hilfe zu tun hat, wird bei uns gerne mit dem roten Kreuz gekennzeichnet, von der Taschenapotheke bis zum Rega-Helikopter. Schon 1977 hat sich aber das amerikanische Rote Kreuz gegen die manchmal ungerechtfertigte Verwendung seines Emblems gewehrt. Deshalb entwarf Leo R. Schwartz, damals Chef des «Emergency Medical Service» der «National Highway Traffic Safety Administration» (NHTSA), den «Star of Life» und liess ihn als geschütztes Markenzeichen registrieren. Das nun auch auf Schweizer Strassen immer häufiger zu sehende Symbol weist den Weg zu qualifizierten 24-Stunden-Notfalldiensten, kennzeichnet deren Fahrzeuge und ziert die Fachzeitschrift für das Personal. Im Zentrum von drei sternförmig angeordneten blauen Balken findet sich der Stab des von Zeus mit einem Blitzschlag exekutierten Heilers Asklepios, umwunden von einer Schlange, die puritanisch sittsam nicht züngelt und so eher einem Regenwurm gleicht. Das erinnert daran, dass auch im Rettungswesen gelegentlich ein Wurm drinsteckt.

Sterne werden für die Qualifizierung von Hotels gut sichtbar angebracht. Die 5-SterneBetriebe bieten den besten Service und/oder fordern die höchsten Preise. Nicht verwunderlich, dass vorgeschlagen wurde, auch an Spitäler und Arztpraxen Sterne zu verleihen. Bei einer durch Soziologen durchgeführten Befragung von 30000 Patienten, die 450 Praxen aufgesucht hatten, erhielten die Berliner Vertragsärzte laut einer im Januar 2000 vorgestellten Studie durchschnittlich 3,3 Sterne. Am häufigsten bekamen die Pulmologen und Urologen 4 oder 5 Sterne, als angenehmste Untersucher erwiesen sich die HNO-Kollegen. Bei einer anderen nachbohrenden Befragung verdienten sich deutsche Zahnärzte durchschnittlich 3,6 Sterne. Wertvoller als die zuerkannten Sterne waren Schwachstellenanalysen, welche aufgrund der Auswertungen für jede Praxis erstellt wurden.

Wie es sich schon in der Hotellerie gezeigt hat, schützen Fixsterne nicht vor schleichenden Qualitätsverlusten. Es wäre auch schwierig, Zimmerkomfort, Verpflegung und medizinisches Angebot in einem Spital nur als Gesamteindruck zu werten. Vielleicht gäbe es *** Ärzte in modernen ${ }^{* * * *}$ Kliniken oder einen jungen ${ }^{* * * * *}$ Chirurgen in einem baulich veralteten
**Landspital. Betriebe mit wenig Sternen hätten nur eine Überlebenschance, wenn sie wie Billigflieger ordentliche Grundleistungen zu stark ermässigten Preisen anbieten könnten. Die ausgesandten Sternforscher wären anfällig für mancherlei Formen der diskreten Bestechung oder Erpressung. Nicht abzusehen die Kosten von Rekursen, mit denen erboste Zurückgesetzte gerichtlich um die verlorene Wertschätzung kämpfen würden.

Als vertrauensbildende Massnahme dienen deshalb weiterhin die behördlich beglaubigten Diplome und Dignitätsatteste, welche nach alter Väter Sitte goldgerahmt an den weissen Wänden der Warteräume befestigt werden. Zudem gibt man den Behandelten immer wieder Gelegenheit, ihre mittlere Zufriedenheit auf umfangreichen Fragebogen anzukreuzen. Offene Quervergleiche zwischen verschiedenen Betrieben werden jedoch datenschützend vermieden, weshalb auch so keine Sterne oder Medaillen zu gewinnen sind.

Zwecks schneller Orientierung der Passanten böte sich eine ganz einfache, rein quantitativ zu begründende Kategorieneinteilung an, eine von weitem erkennbare hellblaue Markierung der verfügbaren Gesundheitsdienste, analog zu den orangen Blickfängern des Grossverteilers:

\begin{tabular}{ll} 
M & ein Mediziner \\
MM & mehrere Medizinerinnen \\
\hline MMM & medizinischer Multimarkt
\end{tabular}

Die vorgeschlagene Regelung ist flexibel, muss doch ein ausgebrannter Einzelkämpfer nur nochmals ein $\mathrm{M}$ an die Fassade kleben, wenn er sich dazu entschliesst, mit einer Kollegin eine Gemeinschaftspraxis aufzunehmen.

Noch ist umstritten, ob die blauen $M M M$ nachts als Leuchtreklame erhellt werden dürfen, obwohl die zugehörigen Praxen von Montag bis Freitag ab 18 Uhr und über das Wochenende geschlossen sind. Das Publikum wird gebeten, sich notfalls an ein öffentliches Spital zu wenden. Wenn die Herberge mit dem grossen $H$ eine Aufnahme herzlos verweigert, folge man dem Star of Life zum Äskulap, wo es klappt. Rund um die Uhr. Bestenfalls. 